\title{
Ten-Day versus 14-Day Levofloxacin-Containing Triple Therapy for Second-Line Anti-Helicobacter pylori Eradication in Taiwan
}

\author{
Wei-Chen Tai, ${ }^{1}$ Chien-Hua Chiu, ${ }^{2}$ Chih-Ming Liang, ${ }^{1}$ Kuo-Chin Chang, \\ Chung-Mou Kuo, ${ }^{1}$ Yi-Chun Chiu, ${ }^{1}$ Keng-Liang Wu, ${ }^{1}$ Ming-Luen Hu, ${ }^{1}$ Yeh-Pin Chou, ${ }^{1}$ \\ Shue-Shian Chiou, ${ }^{1}$ King-Wah Chiu, ${ }^{1}$ Chung-Huang Kuo, ${ }^{1}$ Tsung-Hui Hu, ${ }^{1}$ \\ Ming-Tsung Lin, ${ }^{1}$ and Seng-Kee Chuah ${ }^{1}$ \\ ${ }^{1}$ Division of Gastroenterology, Department of Internal Medicine, Kaohsiung Chang Gung Memorial Hospital, and Chang Gung \\ University College of Medicine, Kaohsiung 833, Taiwan \\ ${ }^{2}$ Division of General Medicine, Department of Internal Medicine, Kaohsiung Chang Gung Memorial Hospital, Kaohsiung 833, Taiwan
}

Correspondence should be addressed to Seng-Kee Chuah; chuahsk@seed.net.tw

Received 14 July 2013; Accepted 5 September 2013

Academic Editor: Chao-Hung Kuo

Copyright (C) 2013 Wei-Chen Tai et al. This is an open access article distributed under the Creative Commons Attribution License, which permits unrestricted use, distribution, and reproduction in any medium, provided the original work is properly cited.

\begin{abstract}
Second-line Helicobacter pylori (H. pylori) eradication with fluoroquinolone-containing triple therapy is one of the recommended treatment options, but neither 7-day nor 10 -day regimens provide $>90 \%$ success rates. The current retrospective study aimed to clarify the effects of 10-day and 14-day levofloxacin-containing triple therapies for second-line $H$. pylori eradication in a Taiwanese cohort and to evaluate the potential clinical factors influencing eradication. A total of 200 patients who failed $H$. pylori eradication using the standard triple therapy were prescribed with either a 10-day (EAL-10) or a 14-day (EAL-14) levofloxacin-containing triple therapy group (levofloxacin $500 \mathrm{mg}$ once daily, amoxicillin $1 \mathrm{~g}$ twice daily, and esomeprazole $40 \mathrm{mg}$ twice daily). Follow-up studies to assess treatment response were carried out 8 weeks later. Eradication rates attained by EAL-10 and EAL-14 were 75.6\%; 95\% $\mathrm{CI}=63.9-85.3 \%$ and $92.5 \%$; $95 \% \mathrm{CI}=84.5-98.1 \%, P=0.002$ in the per protocol analysis and $68 \%$; $95 \% \mathrm{CI}=56.6-78.5 \%$ and $86 \%$; $95 \% \mathrm{CI}=76.8-93.4 \%, P=0.002$ in the intention-to-treat analysis. The duration of $H$. pylori therapy is the independent risk factor of $H$. pylori eradication $(P=0.003)$. In conclusion, 14 -day levofloxacin-containing triple therapy can provide a $>90 \% H$. pylori eradication rate, but 10-day treatment duration may be suboptimal. The longer duration of $H$. pylori therapy (14 days) is the independent risk factor.
\end{abstract}

\section{Introduction}

Many gastrointestinal diseases, either benign or malignant, are associated with Helicobacter pylori (H. pylori) infections, including peptic ulcer diseases, gastric adenocarcinoma, and gastric mucosa-associated lymphoid tissue lymphoma (MALToma) [1-3]. The successful rate of standard firstline triple therapies using a proton pump inhibitor (PPI), clarithromycin, and either amoxicillin or metronidazole for 7 to 14 days has dropped to less than $80 \%$ in many countries especially in areas of high clarithromycin resistance [4-7]. The Maastricht IV/Florence consensus report states that the standard treatment to eradicate $H$. pylori infection is triple therapy; using a proton pump inhibitor (PPI), clarithromycin, and either amoxicillin or metronidazole for 7 to 14 days is recommended for first-line empirical treatment in areas of low clarithromycin resistance, while bismuth-containing quadruple therapy is also an alternative [8]. In areas of high clarithromycin resistance, bismuth-containing quadruple therapies, sequential treatment, or nonbismuth quadruple therapy is used for first-line empirical treatment.

Avoiding problems due to antibiotic resistance has become an important issue when deciding a second-line therapy for H. pylori infection [9-11]. Quinolone has the disadvantage of easily acquired drug resistances [3]. It is therefore an important issue to prescribe it wisely targeting at achieving a high eradication rate. A quinolone-containing triple therapy is recommended by both the Maastricht IV/FlorenceConsensus Report and the second Asia-Pacific Consensus 
Guidelines [8, 12]. However, even large meta-analyses of second-line $H$. pylori eradication with fluoroquinolone-based triple therapy have shown that neither 7 days nor 10 days of therapy provide $90 \%$ or better treatment success [13]. Previous publications in Taiwan used 7-day levofloxacincontaining therapy and attained $75.3-80.3 \%$ of eradication rates $[10,12,14,15]$. However, the reports on second-line eradication by using 14-day levofloxacin-containing triple therapy are few in the literature.

The current retrospective study aimed to clarify the effects of 10-day (EAL-10) and 14-day (EAL-14) esomeprazole/amoxicillin/levofloxacin therapy for patients who failed to have $H$. pylori eradicated after standard triple therapy in Taiwan and to determine the potential clinical factors influencing the eradication.

\section{Materials and Methods}

2.1. Patients. A total of $200 \mathrm{H}$. pylori-infected patients who failed $H$. pylori eradication using the standard triple therapy (PPI twice daily, $500 \mathrm{mg}$ of clarithromycin twice daily, and $1 \mathrm{~g}$ of amoxicillin twice daily) for 7 days were recruited. All the patients were at least 18 years of age and had received endoscopic exam which showed peptic ulcers disease or gastritis. The confirmations of $H$. pylori eradication failure were defined as positive results for both the rapid urease test and histology after first-line eradication. The criteria for exclusion were (a) ingestion of antibiotics, bismuth, or PPI within 4 weeks, (b) allergic history to the medications used, (c) previous gastric surgery, (d) the coexistence of serious concomitant illness (e.g., decompensated liver cirrhosis and uremia), and (e) pregnancy. These 200 patients were prescribed with either a 10-day levofloxacin-containing triple therapy group (levofloxacin $500 \mathrm{mg}$ once daily, amoxicillin $1 \mathrm{~g}$ twice daily, and esomeprazole $40 \mathrm{mg}$ twice daily for 10 days, EAL-10) or a 14-day levofloxacin-containing triple therapy group (EAL-14). Patients were followed up to assess the adverse effects and drug compliance after they finished the medications. All patients received either an endoscopy or a urea breath test eight weeks later. Besides, we also performed a backup urea breath test on all participants to avoid any false-negative results. The definition of poor compliance was that the patient failed to finish $80 \%$ of all medications due to adverse effects $[5,15]$.

This study was approved by both the Institutional Review Board and the Ethics Committee of Chang Gung Memorial Hospital (IRB102-0921B). All patients provided their written informed consent before endoscopic interventions.

2.2. Outcomes. The primary endpoint was the successful eradication of $H$. pylori. There was additional analysis of adverse events during therapy.

\subsection{Diagnosis of Helicobacter pylori Infection}

2.3.1. Rapid Urease Test. The rapid urease test involved the collection of gastric antrum biopsy specimens by endoscopy, which were tested using a urea agar base enriched with $40 \%$ urea solution (eUAB, Oxoid) and a commercial rapid urease test (Pronto Dry, Medical Instrument Corp, Switzerland) [16]. The results of the rapid urease test were interpreted as positive if the color of the gel turned pink or red when examined after $1 \mathrm{~h}$ at room temperature.

2.3.2. Urea Breath Test. The urea breath test was performed according to our previous studies [17]. The cut-off value was set at $4.8 \%$ of $\delta^{13} \mathrm{CO}_{2}$. Staffs were blinded to the Helicobacter pylori status performed to the test.

2.4. Statistical Analysis. The primary outcome variables were the rates of eradication, the presence of adverse events, and the level of patient compliance. Using the SPSS program (Statistical Package for the Social Sciences version 18, Chicago, IL, USA), the chi-square test with or without Yates's correction for continuity and Fisher's exact test were used to compare the major outcomes between groups. Eradication rates were analyzed by both the intention-to-treat (ITT) and per protocol (PP) approach. ITT analysis included all assigned patients who had taken at least one dose of the study medication. Patients whose infection status was unknown following treatment were considered treatment failures for the purposes of the ITT analysis. The PP analysis excluded patients with unknown $H$. pylori status following therapy and those with major protocol violations. A $P$ value of less than 0.05 was considered statistically significant. To determine the independent factors that affected the treatment response, clinical parameters were analyzed by univariate and multivariate analyses.

\section{Results}

A total of 200 patients were enrolled (100 each in the EAL10 and the EAL-14 group). Ten patients lost to follow-up in EAL-10 group and 7 in the EAL-14 group resulted in 90 in the PP study for EAL-10 and 93 for EAL-14 (Figure 1). The demographic data of the two groups are summarized in Table 1, and none of the variables showed significant difference between EAL-10 and EAL-14 groups.

Eradication rates attained by EAL-10 and EAL-14 were 75.6\%; $95 \% \mathrm{CI}=63.9-85.3 \%$ and $92.5 \%$; $95 \% \mathrm{CI}=84.5-98.1 \%$, $P=0.002$ in the PP analysis and 68\%; 95\% CI $=56.6-78.5 \%$ and $86 \%$; $95 \% \mathrm{CI}=76.8-93.4 \%, P=0.002$ in the ITT analysis (Table 2).

3.1. Adverse Events and Complications. The adverse events were $11 \%(11 / 100)$ in EAL-10 group and $16 \%(16 / 100)$ in EAL-14 group (Table 2). These adverse events were abdominal pain, constipation, diarrhea, dizziness, headache, nausea/vomiting, and skin rash, but they were mild and had little disturbance in patients' daily activities (Table 3 ). Both groups had good drug compliances (100\% in EAL-10 group versus $99 \%$ in EAL-14 group).

3.2. Factors Influencing the Efficacy of the Anti-H. pylori Therapies. Univariate analysis showed that the duration of H. pylori eradication $(P=0.002)$ was the clinical factor 


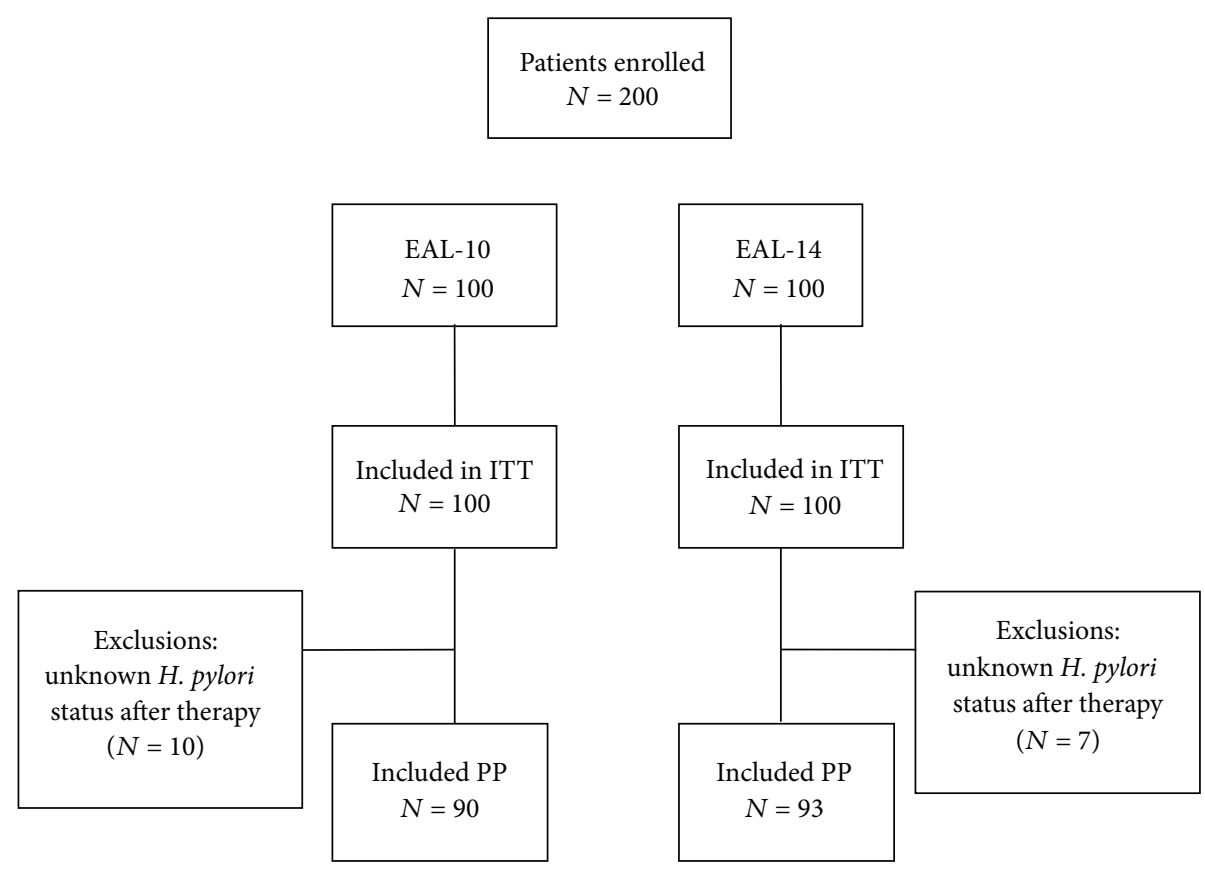

FIGURE 1: Disposition of patients.

TABLE 1: Demographic data and endoscopic appearances of the two patient groups.

\begin{tabular}{lcc}
\hline & EAL-10 $(n=100)$ & EAL-14 $(n=100)$ \\
\hline Characteristics & & \\
Age (year) (mean \pm SD) & $55.6 \pm 13.2$ & $57.6 \pm 12.8$ \\
Gender (male/female) & $44 / 56$ & $45 / 55$ \\
Smoking & $9(9 \%)$ & $6(6 \%)$ \\
Alcohol consumption & $8(8 \%)$ & $13(13 \%)$ \\
Previous history of peptic ulcer & $21(21 \%)$ & $29(29 \%)$ \\
Endoscopic findings & & $35(35 \%)$ \\
Gastric ulcer & $36(36 \%)$ & $20(20 \%)$ \\
Duodenal ulcer & $18(18 \%)$ & $14(14 \%)$ \\
Gastric and duodenal ulcer & $11(11 \%)$ & $31(31 \%)$ \\
Unspecified (include gastritis) & $35(35 \%)$ & \\
\hline
\end{tabular}

TABLE 2: The major outcomes of EAL-10 and EAL-14 eradication therapy.

\begin{tabular}{lccc}
\hline & & Eradication rate & \\
& EAL-10 $(n=100)$ & EAL-14 $(n=100)$ & 0.002 \\
\hline Intention-to-treat & $68 \%(68 / 100)$ & $86 \%(86 / 100)$ & 0.002 \\
Per-protocol & $75.6 \%(68 / 90)$ & $92.5 \%(86 / 93)$ & 0.301 \\
Adverse event & $11 \%(11 / 100)$ & $16 \%(16 / 100)$ & 1.000 \\
Compliance & $100 \%(100 / 100)$ & $99 \%(99 / 100)$ & \\
\hline
\end{tabular}

EAL-10: esomeprazole/amoxicillin/levofloxacin triple therapy $\times 10$ days and EAL-14: esomeprazole/amoxicillin/levofloxacin triple therapy $\times 14$ days.

influencing the efficacy of $H$. pylori eradication therapy (Table 4). Simultaneously, multivariate analysis showed the duration of $H$. pylori eradication (EAL-10 versus EAL-14, OR: 3.98, 95\% CI: $1.60-9.84, P=0.003$ ) was the independent risk factor of successful $H$. pylori eradication (Table 5).

\section{Discussion}

Quinolone-containing triple therapy is one of the recommended second-line therapies after the failure of the standard first-line empirical clarithromycin-containing therapy, with bismuth-containing quadruple therapy as an alternate [8]. 
TABLE 3: Adverse events during EAL-10 and EAL-14 therapies.

\begin{tabular}{lccc}
\hline Adverse events & EAL-10 $(n=100)$ & EAL-14 $(n=100)$ & $P$ value \\
\hline Abdominal pain & 5 & 7 & 0.552 \\
Constipation & 2 & 2 & 1.000 \\
Diarrhoea & 0 & 4 & 0.121 \\
Dizziness & 4 & 1 & 0.369 \\
Headache & 6 & 3 & 0.498 \\
Nausea/vomiting & 2 & 2 & 1.000 \\
Skin rash & 0 & 2 & 0.497 \\
\hline
\end{tabular}

EAL-10: esomeprazole/amoxicillin/levofloxacin triple therapy $\times 10$ days and EAL-14: esomeprazole/amoxicillin/levofloxacin triple therapy $\times 14$ days.

TABLE 4: Univariate analysis of the clinical factors influencing the efficacy of Helicobacter pylori eradication.

\begin{tabular}{lccc}
\hline Principle parameter & Case no. Eradication rate & $P$ value \\
\hline Age & & & \\
$\quad<60$ years & 111 & $82.0 \%(91)$ & 0.318 \\
$\quad \geq 60$ years & 72 & $87.5 \%(63)$ & \\
Sex & & & \\
$\quad$ Female & 98 & $81.6 \%(80)$ & 0.316 \\
$\quad$ Male & 85 & $87.1 \%(74)$ & \\
Smoking & & & \\
$\quad(-)$ & 168 & $83.3 \%(140)$ & 0.472 \\
$\quad(+)$ & 15 & $93.3 \%(14)$ & \\
Previous history of peptic ulcer & & & \\
$\quad(-)$ & 138 & $81.9 \%(113)$ & 0.141 \\
$\quad(+)$ & 45 & $91.1 \%(41)$ & \\
HP eradication (per protocol) & & & \\
$\quad$ EAL-10 & 90 & $75.6 \%(68)$ & 0.002 \\
$\quad$ EAL-14 & 93 & $92.5 \%(86)$ & \\
Compliance & & & - \\
$\quad$ Good & 183 & $84.2 \%(154)$ & \\
$\quad$ Poor & 0 & $0 \%(0)$ & \\
\hline
\end{tabular}

EAL-10: esomeprazole/amoxicillin/levofloxacin triple therapy $\times 10$ days and EAL-14: esomeprazole/amoxicillin/levofloxacin triple therapy $\times 14$ days.

However, we are also aware that bismuth salts are not available in many hospitals. As a matter of fact, such triple therapy with quinolone-containing regimens has been shown to be a good alternative treatment as a second-line $H$. pylori therapy with comparable results to the recommended bismuth-based quadruple therapy [18-20]. Large meta-analyses of secondline $H$. pylori eradication with fluoroquinolone triple therapy have shown that 7 to 10 days of therapy could not provide $90 \%$ or better treatment success $[14,21]$. Published papers on the efficacies of 14-day quinolone-containing triple therapy for second-line therapy are very few, and none of them offered head-to-head data on efficacies for EAL-10 and EAL-14 therapies in similar cohort. This is a very important message because one must target to eradicate the bacteria with a better formula to avoid subsequent quinolone resistance if the eradication failed.
Levofloxacin is a levorotatory isomer of ofloxacin with known activity against many Gram-negative and Grampositive bacteria [22]. The mode of action of levofloxacin is based on the inhibition of bacterial DNA topoisomerase II. A levofloxacin-containing triple therapy is simple and well tolerated and has high compliance (100\% and $99 \%$ in the current study). The relatively low incidence of adverse events among the EAL group was the key factor related to this good compliance. This is important because compliance plays a cardinal role in eradication. In addition, in vitro levofloxacin retains its activity even in dual $H$. pylori resistant strains to clarithromycin and metronidazole [23, 24]. Similar effects have been observed in vivo, showing that most of the dual metronidazole and clarithromycin resistances in $H$. pylori infections are cured with the levofloxacin-containing regimen $[18,25]$. Moreover, there is an in vivo synergistic effect of the quinolone antimicrobial agent and the protonpump inhibitor on strains of $H$. pylori [26].

Drug resistance to antibiotics is an important key factor in successful $H$. pylori eradication. Interestingly, it was just about a decade ago that levofloxacin was chosen as the most promising agent used to overcome antimicrobial resistance among the new antibiotic and drug combinations that had been evaluated, including fluoroquinolones, rifabutin, furazolidone, and azithromycin [27]. Gisbert et al. reported that levofloxacin triple scheme was superior to quadruple therapy ( $81 \%$ versus $70 \%)$ with a lower incidence of side effects (19\% versus $44 \%)$. Again, the 10 -day levofloxacin-based triple scheme was superior to the same 7 -day therapy ( $81 \%$ versus $73 \%$ ) [13]. Today, quinolone resistance is becoming a major concern for the EAL therapy. Just like metronidazole and clarithromycin, drug resistance to levofloxacin is becoming an important factor responsible for unfavorable results. In Taiwan, Kuo et al. reported that levofloxacin-resistant strain was found in $28.3 \%$ of patients [11]. In fact, primary levofloxacin resistance has been increasing in most parts of the world with values of $5.5 \%$ to $32.3 \%$ in countries such as Japan, Brazil, Italy, Hong Kong, and Republic of Korea [28-32]. Therefore, it is very important that the use of quinolone-containing triple therapies need cautious monitoring, because Taiwan is an endemic area for tuberculosis infection [33].

Another reason for the failure of quinolone-containing triple therapies as second-line eradication regimens is the duration of the treatment instead of the dosage. Both the univariate and multivariate analyses in the current study showed that the length of $H$. pylori treatment was the clinical factor influencing the efficacy of eradication. This was similar to Caro and colleagues' report that the duration of treatment is the crucial factor influencing eradication rate but not dosage [20]. In the systemic review reported by Gisbert et al, higher $H$. pylori cure rates with a 10 -day rather than a 7-day regimen were found with the levofloxacin-amoxicillin-PPI combination ( $80 \%$ versus $68 \%$ ), suggesting that the longer (10day) therapeutic scheme should be chosen for levofloxacincontaining triple therapy [13]. In Taiwan, Cheng et al. also reported that prescribing $500 \mathrm{mg}$ and $1000 \mathrm{mg}$ levofloxacin per day did not affect the eradication rates [14]. Liou et al. also attained only $76.9 \%$ eradication with levofloxacin $750 \mathrm{mg}$ once daily [34]. In both studies, the length of treatment 
TABLE 5: Multivariate analysis of the clinical factors influencing the efficacy of Helicobacter pylori eradication.

\begin{tabular}{lccc}
\hline Clinical factor & Coefficient & Standard error & $\begin{array}{c}\text { Odds ratio } \\
(95 \% \text { CI })\end{array}$ \\
\hline $\begin{array}{l}\text { Duration of } \\
\begin{array}{l}\text { H. pylori eradication } \\
\text { (10 days versus 14 days) }\end{array}\end{array}$ & 1.38 & 0.46 & 3.98 \\
\hline
\end{tabular}

EAL-10: esomeprazole/amoxicillin/levofloxacin triple therapy $\times 10$ days and EAL-14: esomeprazole/amoxicillin/levofloxacin triple therapy $\times 14$ days.

was only 7 days. The current study showed that EAL-10 could be suboptimal and only attained $75.6 \%$ eradication rate in the PP analysis. None of these studies with 7-10 days of levofloxacin-containing triple therapies attained $90 \%$ or better treatment success. Two recently published studies reported that extending the length of quinolone-containing triple therapies to 14 days could achieve eradication success up to $95 \%$ (moxifloxacin) and 93.6\% (levofloxacin) $[35,36]$. Consequently, the current study achieved an eradication rate of $92.5 \%$ in the EAL-14 group in PP analysis but only $75.6 \%$ in the EAL-10 group. Our study result adds on a potentially important message that 14 days should be the optimal length of treatment for quinolone-containing triple therapies as a second-line $H$. pylori treatment option instead of the 7-10day regimens. The bottom line is that quinolone resistance is carefully monitored.

However, the current study encountered its limitations. First, since our laboratory could not perform CYP2C19 genotyping, we used an esomeprazole-based regimen because it had minimal first-pass metabolism and had a greater gastric acid suppression effect than omeprazole. Second, there was the lack of information regarding the prevalence of antimicrobial resistance.

\section{Conclusions}

A 14-day levofloxacin-containing triple therapy can provide a $>90 \%$ H. Pylori eradication rate, but 10 -day treatment duration may be suboptimal. The longer duration is the independent risk factor for eradication. This is a very important message since quinolone easily acquires resistance. Meanwhile, a continuous search for novel second-line therapeutic approaches which are cost-effective and minimize drug resistance to cure $H$. pylori infection is still mandatory.

\section{Conflict of Interests}

The authors declare that they received no current external funding sources for this study.

\section{Authors' Contribution}

Wei-Chen Tai and Chien-Hua Chiu contributed equally to the work.

\section{Acknowledgments}

The authors would like to acknowledge Miss Chih-Yun Lin for her assistance in the statistical analysis and Chang Gung
Medical Foundation, Kaohsiung Chang Gung Memorial Hospital Tissue Bank (CLRPG870463) for technical support. Wei-Chen Tai and Chien-Hua Chiu are co-first authors.

\section{References}

[1] D. Y. Graham, G. M. Lew, P. D. Klein et al., "Effect of treatment of Helicobacter pylori infection on the long-term recurrence of gastric or duodenal ulcer. A randomized, controlled study," Annals of Internal Medicine, vol. 116, no. 9, pp. 705-708, 1992.

[2] S. K. Chuah, F. W. Tsay, P. I. Hsu, and D. C. Wu, "A new look at anti-Helicobacter pylori therapy," World Journal of Gastroenterology, vol. 17, no. 35, pp. 3971-3975, 2011.

[3] C. H. Kuo, P. I. Hsu, F. C. Kuo et al., "Comparison of 10 day bismuth quadruple therapy with high-dose metronidazole or levofloxacin for second-line Helicobacter pylori therapy: a randomized controlled trial," Journal of Antimicrobial Chemother$a p y$, vol. 68, pp. 222-228, 2013.

[4] P. I. Hsu, D. C. Wu, J. Y. Wu, and D. Y. Graham, "Is there a benefit to extending the duration of Helicobacter pylori sequential therapy to 14 days?” Helicobacter, vol. 16, no. 2, pp. 146-152, 2011.

[5] D. Y. Graham and L. Fischbach, "Helicobacter pylori treatment in the era of increasing antibiotic resistance," Gut, vol. 59, no. 8, pp. 1143-1153, 2010.

[6] D. Y. Graham, H. Lu, and Y. Yamaoka, "A report card to grade Helicobacter pylori therapy," Helicobacter, vol. 12, no. 4, pp. 275278, 2007.

[7] Y. Yamaoka, D. Y. Graham, and H. Lu, "Should triple therapy for Helicobacter pylori infection be abandoned as no longer effective?" The American Journal of Gastroenterology, vol. 4, pp. 65-67, 2008.

[8] P. Malfertheiner, F. Megraud, C. A. O’Morain et al., "Management of Helicobacter pylori infection-the Maastricht IV/ Florence Consensus Report," Gut, vol. 61, no. 5, pp. 646-664, 2012.

[9] T. H. Hu, S. K. Chuah, P. I. Hsu et al., "Randomized comparison of two nonbismuth-containing rescue therapies for Helicobacter pylori," The American Journal of the Medical Sciences, vol. 342, no. 3, pp. 177-181, 2011.

[10] P. I. Hsu, D. C. Wu, J. Y. Wu, and D. Y. Graham, "Modified sequential Helicobacter pylori therapy: proton pump inhibitor and amoxicillin for 14 days with clarithromycin and metronidazole added as a quadruple (hybrid) therapy for the final 7 Days," Helicobacter, vol. 16, no. 2, pp. 139-145, 2011.

[11] C. H. Kuo, H. M. Hu, F. C. Kuo et al., "Efficacy of levofloxacinbased rescue therapy for Helicobacter pylori infection after standard triple therapy: a randomized controlled trial," Journal of Antimicrobial Chemotherapy, vol. 63, no. 5, pp. 1017-1024, 2009.

[12] K. M. Fock, P. Katelaris, K. Sugano et al., "Second AsiaPacific Conference. Second Asia-Pacific Consensus Guidelines 
for Helicobacter pylori infection," Journal of Gastroenterology and Hepatology, vol. 24, no. 10, pp. 1587-1600, 2009.

[13] J. P. Gisbert and F. De La Morena, "Systematic review and metaanalysis: levofloxacin-based rescue regimens after Helicobacter pylori treatment failure," Alimentary Pharmacology and Therapeutics, vol. 23, no. 1, pp. 35-44, 2006.

[14] H. C. Cheng, W. L. Chang, W. Y. Chen, H. B. Yang, J. J. Wu, and B. S. Sheu, "Levofloxacin-containing triple therapy to eradicate the persistent $H$. pylori after a failed conventional triple therapy," Helicobacter, vol. 12, no. 4, pp. 359-363, 2007.

[15] S. K. Chuah, P. I. Hsu, K. C. Chang et al., "Randomized comparison of two non-bismuth-containing second-line rescue therapies for Helicobacter pylori," Helicobacter, vol. 17, no. 3, pp. 216-223, 2012.

[16] J. Yakoob, S. Abid, W. Jafri, Z. Abbas, M. Islam, and Z. Ahmad, "Comparison of biopsy-based methods for the detection of Helicobacter pylori infection," British Journal of Biomedical Science, vol. 63, no. 4, pp. 159-162, 2006.

[17] N. J. Peng, K. H. Lai, R. S. Liu et al., "Clinical significance of oral urease in diagnosis of $H$. pylori infection by 13C-urea breath test," Digestive Diseases and Sciences, vol. 46, no. 8, pp. 17721778, 2001.

[18] C. Bilardi, P. Dulbecco, P. Zentilin et al., "A 10-day levofloxacinbased therapy in patients with resistant Helicobacter pylori infection: a controlled trial," Clinical Gastroenterology and Hepatology, vol. 2, no. 11, pp. 997-1002, 2004.

[19] R. W. Schrauwen, M. J. Janssen, and W. A. de Boer, "Sevenday PPI-triple therapy with levofloxacin is very effective for Helicobacter pylori eradication," The Netherlands Journal of Medicine, vol. 67, no. 3, pp. 96-101, 2009.

[20] S. di Caro, F. Franceschi, A. Mariani et al., "Second-line levofloxacin-based triple schemes for Helicobacter pylori eradication," Digestive and Liver Disease, vol. 41, no. 7, pp. 480-485, 2009.

[21] J. P. Gisbert, A. Pérez-Aisa, F. Bermejo et al., "Second-line therapy with levofloxacin after failure of treatment to eradicate Helicobacter pylori infection: time trends in a Spanish multicenter study of 1000 patients," Journal of Clinical Gastroenterology, vol. 47, pp. 130-135, 2013.

[22] P. I. Hsu, K. H. Lai, H. H. Tseng et al., "Eradication of Helicobacter pylori prevents ulcer development in patients with ulcer-like functional dyspepsia," Alimentary Pharmacology and Therapeutics, vol. 15, no. 2, pp. 195-201, 2001.

[23] J. Yahav, H. Shmuely, Y. Niv, J. Bechor, and Z. Samra, "In vitro activity of levofloxacin against Helicobacter pylori isolates from patients after treatment failure," Diagnostic Microbiology and Infectious Disease, vol. 55, no. 1, pp. 81-83, 2006.

[24] D. Antos, W. Schneider-Brachert, E. Bästlein et al., "7-day triple therapy of Helicobacter pylori infection with levofloxacin, amoxicillin, and high-dose esomeprazole in patients with known antimicrobial sensitivity," Helicobacter, vol. 11, no. 1, pp. 39-45, 2006.

[25] J. P. Gisbert, “"Rescue” regimens after Helicobacter pylori treatment failure," World Journal of Gastroenterology, vol. 14, no. 35, pp. 5385-5402, 2008.

[26] M. Tanaka, E. Isogai, H. Isogai et al., "Synergic effect of quinolone antibacterial agents and proton pump inhibitors on Helicobacter pylori," Journal of Antimicrobial Chemotherapy, vol. 49, no. 6, pp. 1039-1040, 2002.

[27] M. Guslandi, "Review article: alternative antibacterial agents for Helicobacter pylori eradication," Alimentary Pharmacology and Therapeutics, vol. 15, no. 10, pp. 1543-1547, 2001.
[28] Y. Matsumoto, I. Miki, N. Aoyama et al., "Levofloxacin-versus metronidazole-based rescue therapy for $H$. pylori infection in Japan," Digestive and Liver Disease, vol. 37, no. 11, pp. 821-825, 2005.

[29] L. G. Coelho, L. D. Moretzsohn, W. L. Vieira et al., "New oncedaily, highly effective rescue triple therapy after multiple Helicobacter pylori treatment failures: a pilot study," Alimentary Pharmacology and Therapeutics, vol. 21, no. 6, pp. 783-787, 2005.

[30] L. Marzio, D. Coraggio, S. Capodicasa, L. Grossi, and G. Cappello, "Role of the preliminary susceptibility testing for initial and after failed therapy of Helicobacter pylori infection with levofloxacin, amoxicillin, and esomeprazole," Helicobacter, vol. 11, no. 4, pp. 237-242, 2006.

[31] W. M. Wong, Q. Gu, K. M. Chu et al., "Lansoprazole, levofloxacin and amoxicillin triple therapy versus quadruple therapy as second-line treatment of resistant Helicobacter pylori infection," Alimentary Pharmacology and Therapeutics, vol. 23, no. 3, pp. 421-427, 2006.

[32] J. M. Kim, J. S. Kim, N. Kim, S. G. Kim, H. C. Jung, and I. S. Song, "Comparison of primary and secondary antimicrobial minimum inhibitory concentrations for Helicobacter pylori isolated from Korean patients," International Journal of Antimicrobial Agents, vol. 28, no. 1, pp. 6-13, 2006.

[33] W. C. Tai, T. H. Hu, C. H. Lee, H.-H. Chen, C. C. Huang, and S.-K. Chuah, "Ano-perianal tuberculosis: 15 years of clinical experiences in Southern Taiwan," Colorectal Disease, vol. 12, no. 7, pp. el14-e120, 2010.

[34] J. M. Liou, C. C. Chen, M. J. Chen et al., "Empirical modified sequential therapy containing levofloxacin and high-dose esomeprazole in second-line therapy for Helicobacter pylori infection: a multicentre clinical trial," Journal of Antimicrobial Chemotherapy, vol. 66, no. 8, pp. 1847-1852, 2011.

[35] S. Miehlke, S. Krasz, W. Schneider-Brachert et al., "Randomized trial on 14 versus 7 days of esomeprazole, moxifloxacin, and amoxicillin for second-line or rescue treatment of Helicobacter pylori infection," Helicobacter, vol. 16, no. 6, pp. 420-426, 2011.

[36] S. K. Chuah, W. C. Tai, P. I. Hsu et al., "The efficacy of secondline anti-Helicobacter pylori therapy using an extended 14days levofloxacin/amoxicillin/protonpumpinhibitors-a pilot study," Helicobacter, vol. 17, pp. 374-381, 2012. 


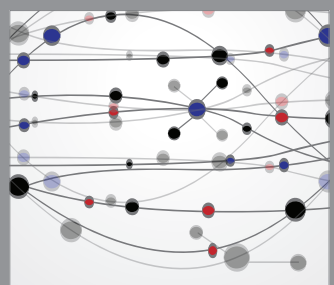

The Scientific World Journal
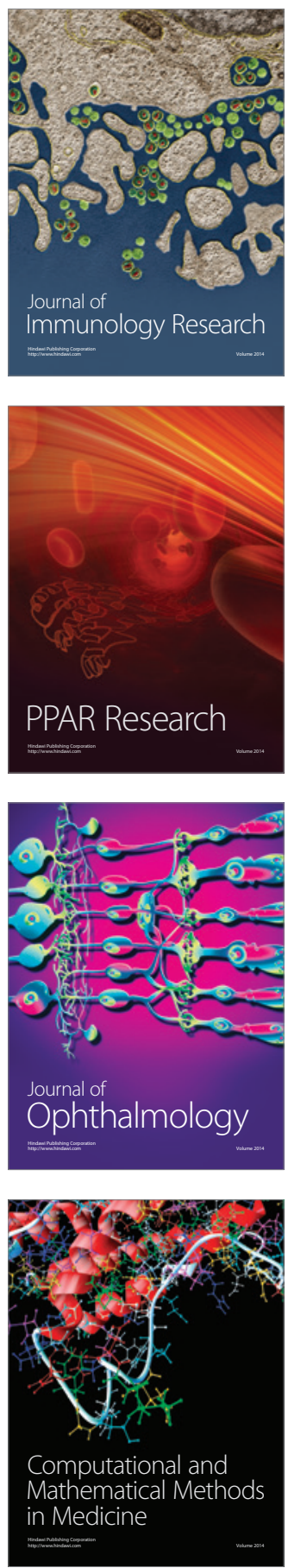

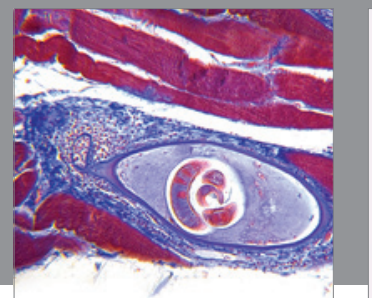

Gastroenterology

Research and Practice
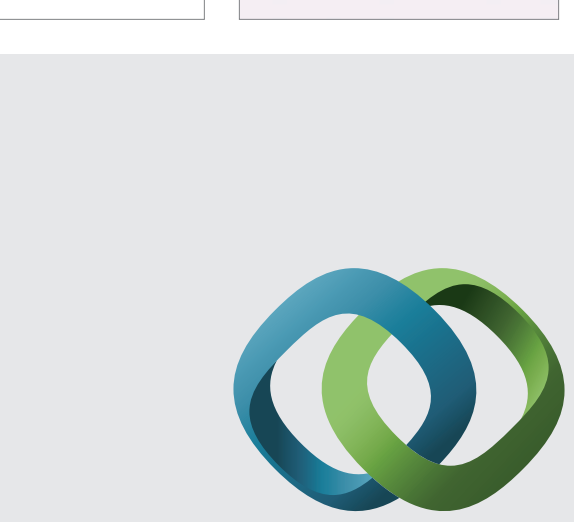

\section{Hindawi}

Submit your manuscripts at

http://www.hindawi.com
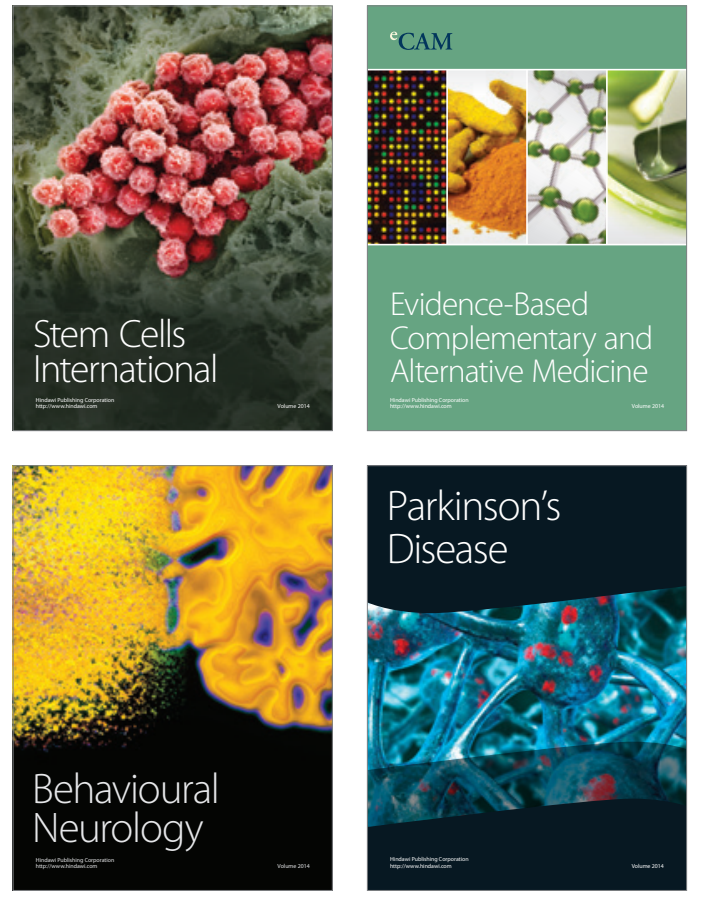
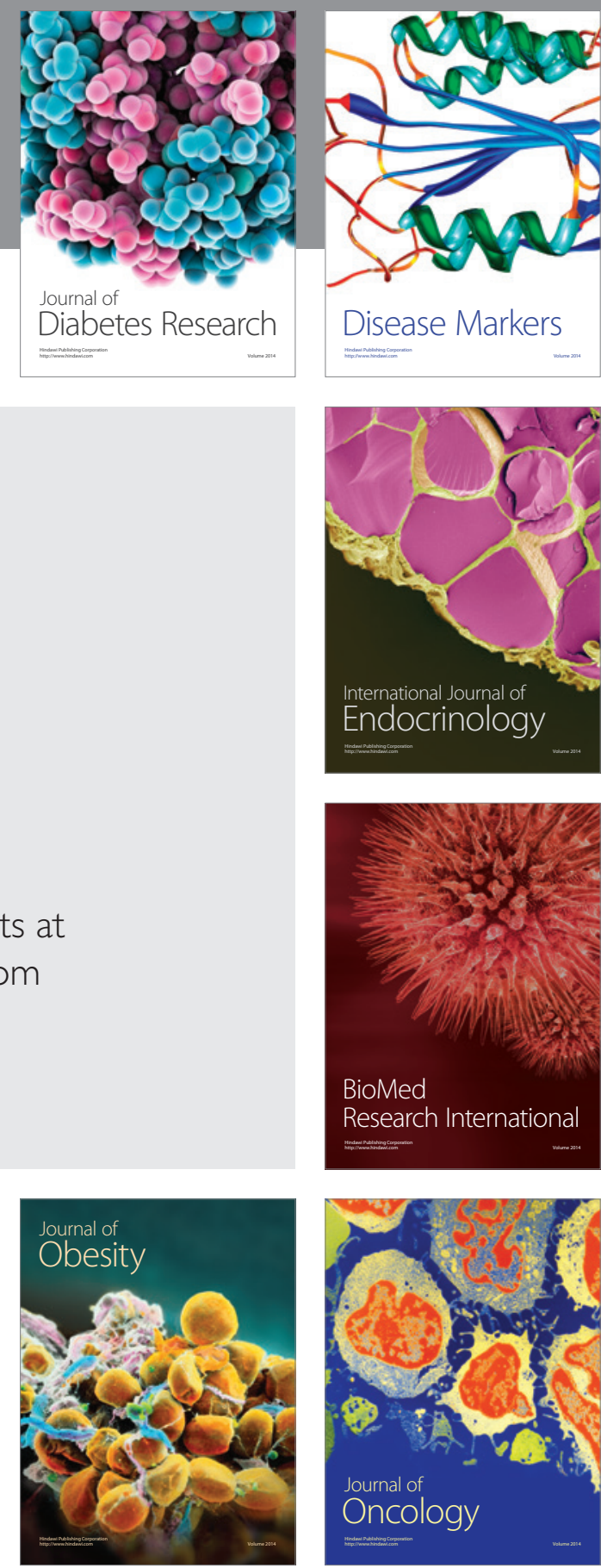

Disease Markers
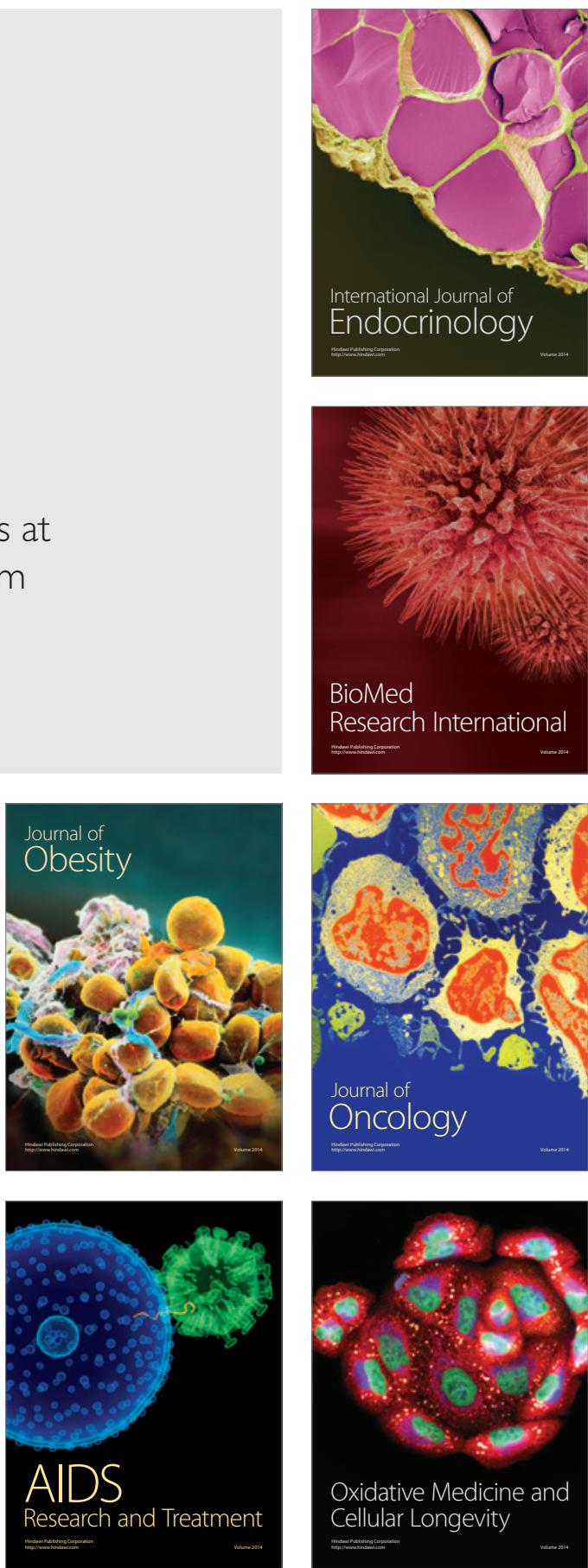